\title{
FACSIMILE TRANSMISSION
}

$\mathrm{A}^{\mathrm{N}}$ $\mathrm{N}$ instructive paper, by $\mathrm{G}$. Herrick, describing the progress in the United States of facsimile transmission, that is, the transmission of the printed page, drawings, photographs, documents and original messages by radio or wire telegraphy, appears in the Electrical Review of January 19. Certain electrical problems, more commercial than technical, have yet to be solved, and further development makes it necessary to use the best type of paper for recording purposes. Recently when President Roosevelt went to Canadian waters on a fishing trip, on board the U.S. Cruiser Tuscaloosa, he received a total of about 50,000 words of news on a facsimile receiver in his office-cabin, while the ship's officers received the same news on a duplicate receiver in the vessel's office.

The information transmitted was gathered by Trans-Radio News, set in type by the Koppel Printing Co. in New York, and broadcast from Cartaret, N.J., by the Finch system of transmission over the regular $780 \mathrm{kc}$. used by the station WOR of the Mutual Broadcasting Co. The transmissions were received in the afternoon in the Tuscaloosa, which reached a maximum distance of 650 miles from the transmitter. As the ship returned and neared New York, the smaller transmitter at the Finch Telecommunications Laboratories in Broadway, New York, took over transmission. For the transmission, alternating current at 110 volts and 60 cycles was used to energize both the transmitter and receiver. One of the difficulties to be overcome in the development of facsimile transmission is the fact that the power stations in different States often vary in frequency.

At present three systems of facsimile transmission of printed material and pictures are being developed. The R.C.A. system is controlled by the Radio Corporation of America and uses what is called 'percussion' recording through varying electrical impulses, which record lines on any grade of paper through the medium of a carbon sheet. The drawback is that it operates at only about three feet an hour.

The system controlled by Finch Telecommunication Laboratories records on special paper that is basically black and coated with an 'eggshell' surface. Across this an electric stylus travels, being regulated by impulses received at standard radio frequencies, or over a telegraph or telephone wire. The stylus draws a hundred lines to the inch and, by oxidizing the surface coating to a greater or lesser degree, records a variety of shades from grey to black.

The system of J. V. L. Hogan, consulting engineer of New York, was used with interesting results at the Toronto Fair by the Globe-Mail of that city. This method also employs the stylus principle of recording. Instead of oxidizing a specially coated paper, a series of points that are in contact with specially sensitized paper in rolls delivers varying electrical impulses to the paper and develops the required type of picture as the paper unrolls in the receiver. This system is extremely clear and can record very minute details, such as a photograph of a lace tablecloth or type down to 6-point. All the three differing systems require the material to be transmitted to be placed on a revolving drum, so that it is scanned by a point of light with which is associated a photo-electric tube that reacts to the reflections of light from light and dark substances, varying the impulses sent out.

In the United States, development of this kind of transmission has been delayed by the public and technical interest taken in television. To-day, with television making satisfactory progress, facsimile transmission is being developed rapidly. What the public want is low-cost synchronizers which are both efficient and do not slow down transmission. Some of the synchronizers in use necessitate low speed. The transmissions to the Tuscaloosa, for example, were received at three feet an hour.

Photographs both by radio and wire are being sent across the Atlantic at the present time. As an indication of future possibilities, consider the dissemination of news from one city to another by transmitting entire printed pages with illustrations, which would be multiplied by photo-offset printing, and could be sold in the streets within two hours after being sent. This plan envisages the use of wired rather than wireless transmission; technicians engaged in facsimile development suggest that their experiments have indicated that wired facsimile may be less certain than wireless, except in certain sections.

Unlike the television receiver, facsimile apparatus can be built at low cost because it is comparatively simple. The cheapest television sets in the United States cost about $£ 39$, but it is believed that a standard facsimile receiver could be constructed to retail at $£ 10$.

The Finch organization has recently brought out mobile facsimile transmitters and receivers. Several police motor-cars in Bergen County, New Jersey, just across the Hudson River from New York City, have been equipped with facsimile two-way units that can send and receive alternately. A New York newspaper is experimenting with a motor-car into which is built transmitter, receiver and a dark room. It has a tiny writing space and typewriter for the news man.

The Finch Company has developed a method of utilizing any telephone; strangely enough, the method has been approved by the telephone company, which has rigorous rules against tampering with its lines or equipment. The unit is an inductive coupling and cable. With one end of the cable attached to the transmitting and receiving unit in the motor-car or special truck, the other end of the cable is carried from the kerb into the nearest public or other telephone. The user then calls the telephone operator and says, "Do not interrupt, this wire is being used for a facsimile transmission". The use is thereafter charged at the ordinary telephone rate. During the transmission of news and pictures in this way, it is not necessary to use the telephone, as the office of reception can transmit messages of instruction back to the truck transmitter by writing and having them scanned on its own transmitting drum.

Similar testing of facsimile is being done by the U.S. Army, and the Navy is understood to be increasingly interested in possible use of facsimile for intercommunication between naval ships and messages to and from bases. 